\title{
The effect of asymmetric reinforcement on curved bridge
}

\author{
Dai Fan ${ }^{1, ~ a}$, He Jian $^{1, b}$, You Shangyou ${ }^{1, ~ c}$, Luo Muxin², d \\ ${ }^{1}$ Zhanjiang City Highway Authority, Zhanjiang ,Guangdong 524043, China \\ ${ }^{2}$ College of Science and Engineering, Jinan University, Guangzhou, Guangdong 510000, China \\ adaifan666@163.com, ${ }^{\text {bjjk1206@126.com, }{ }^{c} 191493928 @ q q . c o m ~}{ }^{d}$ mxluode@163.com (corres- \\ ponding author)
}

KEYWORD: asymmetric reinforcement; curved bridge; torsion.

ABSTRACT: Due to their geometry, continuous curved bridge under gravity load will produce torque, which danger to bridge structure. In order to reduce the weight of load torque, with the method of asymmetric reinforcement, this paper introduces the three forms of prestressed reinforcement, through optimizing combination, to find the best arrangement of prestressed to adjust torque.

\section{INTRODUCTION}

Recent years, with the rapid development of China's high-grade highways and urban roads, overpasses and flyovers growing number because of the terrain, the overall linear route continuity and urban greening and other issues, all need the curve layout. Curved Bridge because of its curved shape, more fully meet the design requirements, thus gradually been widely used ${ }^{[1,2,3]}$. In the field of bridge engineering, especially those with many curved bridge in the city, in order to meet the requirements of the bridge alignment and appearance often choose curved bridge ${ }^{[4]}$. In the curved bridge structure, either in static loads or moving loads, bending moments and torque coupling phenomenon is very prominent. ${ }^{[5]}$.

XiaoDongJiang Bridge, the plane is located on the circular curve $\mathrm{R}=400 \mathrm{~m}$, the bridge across combination is: $36+60+36$ m prestressed concrete continuous box girder bridge. ( Fig.1).
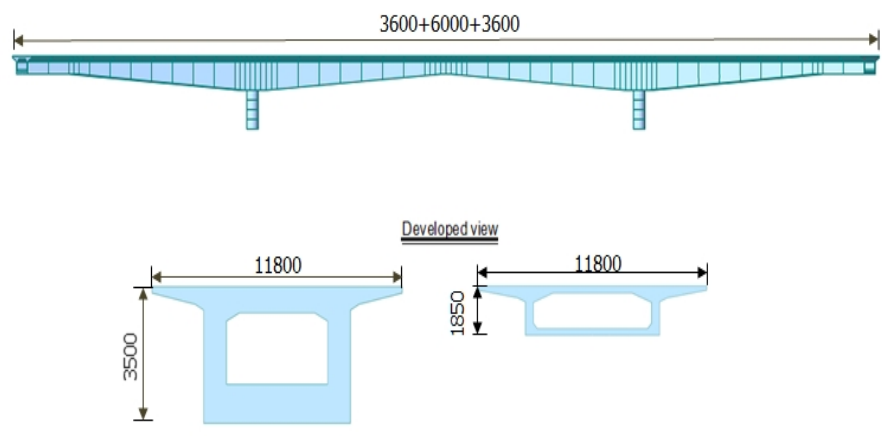

Cross-Section of the box-girder

Fig.1 General view of curved continuous box-girder bridge

\section{Research method:}

Pre-stressed curved bridge under gravity load will produce torque, because the curved bridge with curved geometry itself, when setting up support, two bearings in the plane of the attachment you can see the weight of partial load of the curved bridge (figure 2), of the part on the top of the dotted line to produce out of trend, to various trend below the dotted line. Bridge in the general design, a structure of crossing the river, on both sides of the line to consider, also want to consider the water of the ship navigation problems, so the radius of curvature and span, are chosen to reduce the bending of the bridge under dead load torque, to consider the structure from itself. Specific methods can be used in a torsional support eccentric and asymmetry prestressed reinforcement method. Now to the asymmetry of prestressed reinforcement to the effects of the curved bridge torque make specific discussion. 


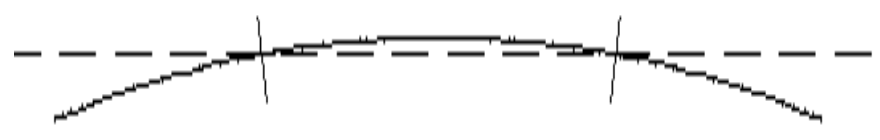

Fig 2 Deviation of deadweight

The fundamental purpose of this study is to present the effect of the radius of curvature on seismic response of curved bridge, so a few models with different radius of curvature are built by using the finite element software Midas Civil.

The main girder, piers and abutments are modeled as 3D beam elements. The nodes at the bottom of piers are given the boundary condition of fixity. Full-bridge model of Plane Frame is calculated by using finite element analysis method to simplify the structure of the full-bridge 93 nodes and 70 units in which the main beam node number is 1 to 63 , the corresponding unit number is 1 to 62 . Pier node number is 64 to 74, corresponding to the unit number is 63 to 71 .

General prestressed bridge of prestressed steel beam shapes can be divided into three types, corresponding to three directions of the prestressed, convenient for later described, I will be in figure 2 a. Known as the straight steel beam, c.called flat bending steel beam, b. called vertical bengding steel beam. As shown in figure 3 .

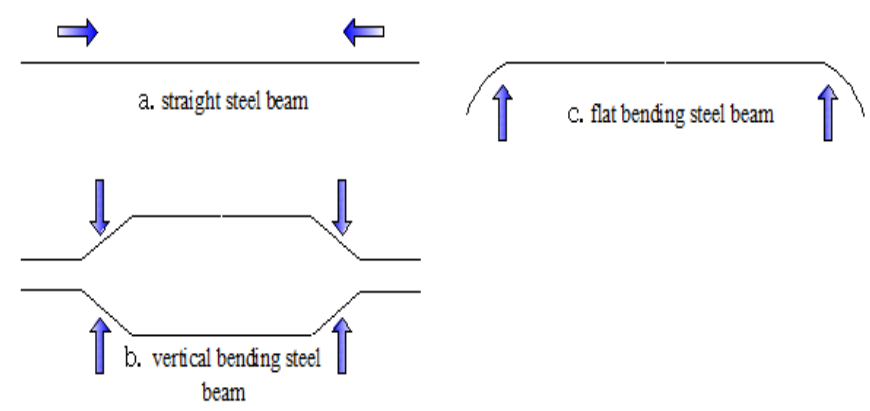

Fig 3 Three kinds of prestressed steel beam

To the flat bending steel beam and vertical bending steel beam, as shown in figure 3, vertical bending steel beam in addition to ele vated to component and upward force, they will be in the cross section form a pair of equal symmetry. By the same token, the flat bending steel beam will be formed in the cross section of a pair of equal force of symmetry. At the time of the two groups of symmetrical equals the force, they will not produce additional torque to cross section, when the force is not equal to the two groups, they would have a corresponding torque of cross section, take advantage of this phenomenon, which can be used to reduce the torque generated by the dead weight.

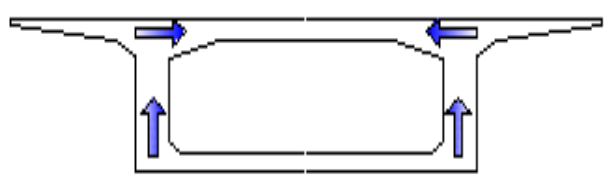

Figure 4 section force direction

Curved bridge under the action of gravity will produce torque, to resist under the weight of the torque, the decrease on the left side of the force, increase the symmetry of the force on the right(figure 4). Don't change to bending moment and the effect of prestress of ele vated while minimize a percentage, on the other side of the same percentage increase. First is the vertical steel beam bending peace, adopt to reduce $30 \%$ on the left side of the steel beam tension, $30 \%$ increase on the right side of the steel beam. That although most can reduce the torque generated by the self weight, but also will increase the corresponding bending bridge cross the bridge to the torque, especially in the bearing. For this, the support using increased by $30 \%$ on the left side of straight line steel beam, the $30 \%$ reduction on the right side of the straight line of steel beam, due to the linear steel beam in 
the bearing is shorter, less affected by the curvature of curved bridge, and no geometric moment itself, it can significantly offset by vertical bending cross the bridge to the bending moment increased. In addition, the outside support, can use flat bar bending to adjust. Concrete method is to add 2 steel beam, reduce the no. 3 steel beam (FIG. 5), the role of both to achieve adjusting torque, also can have cut changed from vertical bending cross the bridge to the bending moment increased.

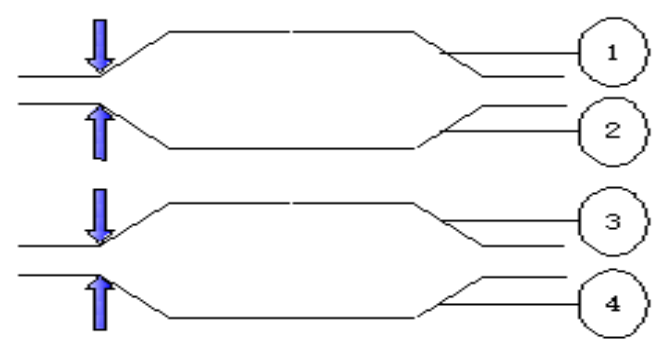

Fig 5 vertical bending steel beamText and indenting

Table 1 asymmetry reinforcement to adjust the configuration of the first scheme

\begin{tabular}{ccccccccc}
\hline & \multicolumn{1}{c}{$\begin{array}{c}\text { vertical bengding steel } \\
\text { beam }\end{array}$} & $\begin{array}{c}\text { straight } \\
\text { steel } \\
\text { beam }\end{array}$ & & & & & \\
flat bending steel beam \\
first & $\mathrm{F} 1$ & $\mathrm{~F} 2$ & $\mathrm{~F} 3$ & $\mathrm{~T} 1$ & $\mathrm{~T} 2$ & $\mathrm{~T} 3$ & $\mathrm{~T} 4$ & $\mathrm{~T} 5$ \\
sche & $50 \%$ & $40 \%$ & $30 \%$ & $50 \%$ & $40 \%$ & $30 \%$ & $20 \%$ & $10 \%$ \\
me & $\mathrm{F} 4$ & $\mathrm{~F} 5$ & & & $\mathrm{~T} 6$ & $\mathrm{~T} 7$ & $\mathrm{~T} 8$ & \\
& $20 \%$ & $10 \%$ & & & $0 \%$ & $0 \%$ & $0 \%$ & \\
\hline
\end{tabular}

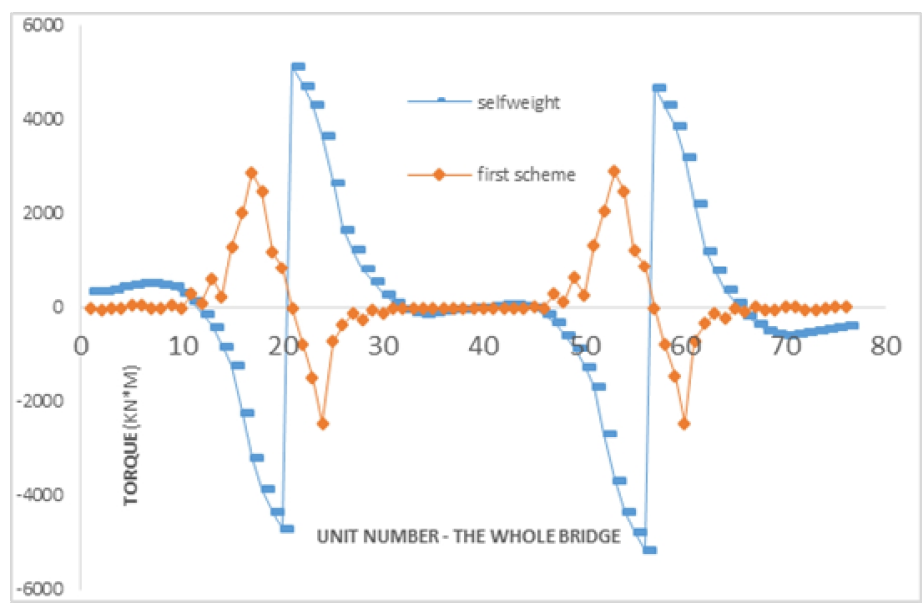

Fig 6 The first scheme of torque to improve

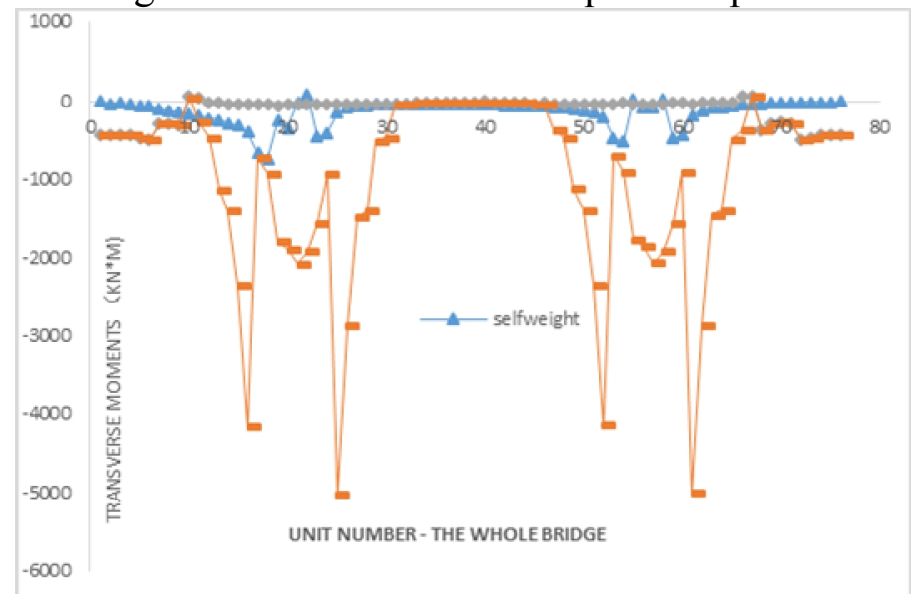

Fig 7 The first scheme of torque to improve 
The figure 6 shows that the first scheme of torque to reduce obviously, but from the figure 7 shows the bridge to the improvement of the transverse moment is not good enough, most of the bridge to the bottom of the transverse moment value is bigger, the next step adjustment scheme adopts the erected reinforced unchanged, change the flat steel bending beam. The bridge to the transverse moment in the vicinity of bearing has been reduced, so the focus adjustment on either side of the support area to cross the bridge to the bending moment. Keeps erect reinforcement and linear constant, increase a pair of T6 adjustment of $20 \%$, the T5 changed from $10 \%$ to $20 \%$, the specific numerical see table 2 . The second scheme improved the torque figure and the difference is not big, cross the bridge to the bending moment to improve is bigger, specific see chart 8 .

Table 2 asymmetry reinforcement to adjust the configuration of the second scheme

\begin{tabular}{|c|c|c|c|c|c|c|c|c|}
\hline & \multicolumn{3}{|c|}{$\begin{array}{l}\text { vertical bengding steel } \\
\text { beam }\end{array}$} & \multirow{2}{*}{$\begin{array}{c}\begin{array}{c}\text { straight } \\
\text { steel beam }\end{array} \\
\mathrm{T} 1\end{array}$} & \multicolumn{4}{|c|}{ flat bending steel beam } \\
\hline \multirow{4}{*}{$\begin{array}{c}\text { the } \\
\text { second } \\
\text { scheme }\end{array}$} & F1 & F2 & F3 & & $\mathrm{T} 2$ & T3 & $\mathrm{T} 4$ & T5 \\
\hline & $50 \%$ & $40 \%$ & $30 \%$ & $50 \%$ & $40 \%$ & $30 \%$ & $30 \%$ & $20 \%$ \\
\hline & $\mathrm{F} 4$ & F5 & & & T6 & $\mathrm{T} 7$ & $\mathrm{~T} 8$ & \\
\hline & $20 \%$ & $10 \%$ & & & $20 \%$ & $0 \%$ & $0 \%$ & \\
\hline
\end{tabular}

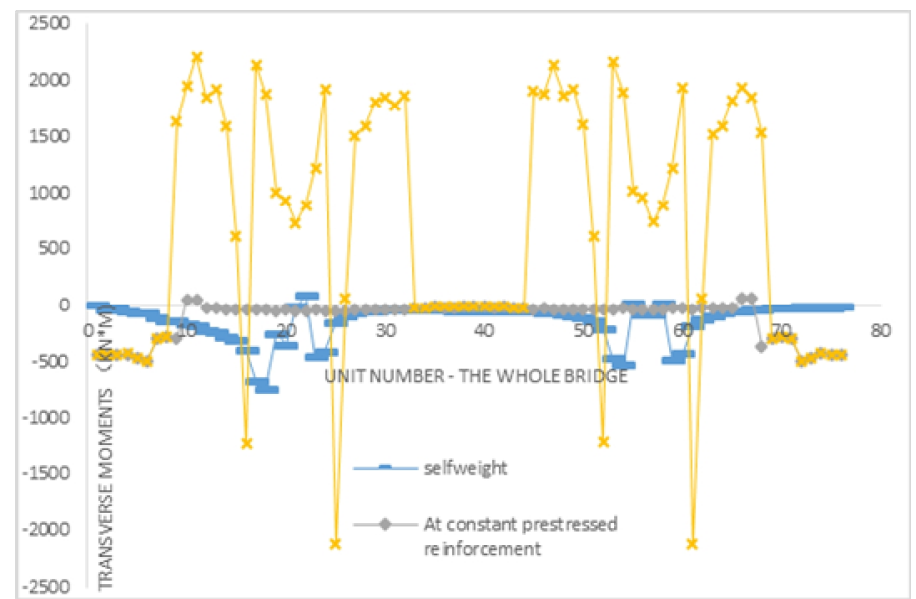

Fig 8 The scheme of transverse moment to improves

The figure 8 shows the second plan compared to the first bridge to the transverse moment integral lifting upwards, plus or minus maximum between $(2000 \sim 2000)$, maximum/small distribution is more reasonable.

After the discussion of the different schemes, it is concluded that the asymmetric adjustment of some characteristics of the prestressed reinforcement, listed as follows:

1. Vertical steel beam due to its larger more than the vertical bending flat steel bending beam can improve the output torque of the curved bridge.

2. Vertical steel beam bridge in improving the bending torque at the same time, also can produce a substantial the bridge to the transverse moment.

3. Horizontal bending steel beam on the improvement of the curved bridge torque effect is not obvious, but it will produce more apparent to the bending moment and transverse bridge for vertical steel beam transverse bridge because of the improved torque to the bending moment can have very good cutting effect.

4. Asymmetric side to reduce prestress on the other side to increase the same numerical prestressed won't cause an effect the bridge to the bending moment.

\section{Conclusions}

Asymmetry reinforcement was studied on the influence of curved bridge, and according to the characteristics of the curved bridge under the action of dead load to produce torque pointed proposed 
asymmetric adjustment of prestressed composite, to cut the curved bridge under the action of dead load torque have a significant effect. Specific what time of the conclusion:

1 , the vertical bending srteel beam tension on the left to reduce by $30 \%$, a $30 \%$ increase in the right, can significantly improve the torque under the action of gravity; And the adjustment of the flat bar bending also leads to the improvement of the torque (figure 9).

2, due to the decrease of the vertical bending srteel beam tension on the left, leading to curved bridge cross the bridge to the bending moment on the left side of bending moment increase, increasing the order of magnitude is not big. In addition, if we can only reduce the flat bar bending tensile strength of the no. 1,2, and increase the tensile strength of the no. 3, 4, while brings the improvement of the torque, but also with the same order of magnitude of the transverse bridge to the increase of bending moment. (figure 10)

3 , adjusted straight srteel beam and flat bending srteel beam of reinforcement can effectively offset changed from vertical bending cross the bridge to the bending moment increased. (figure 11)

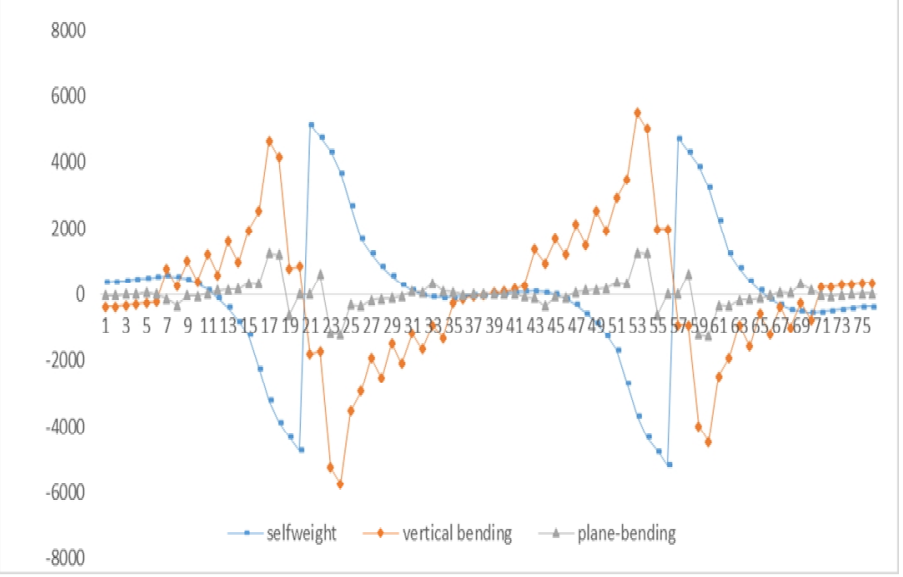

Fig 9 Torque

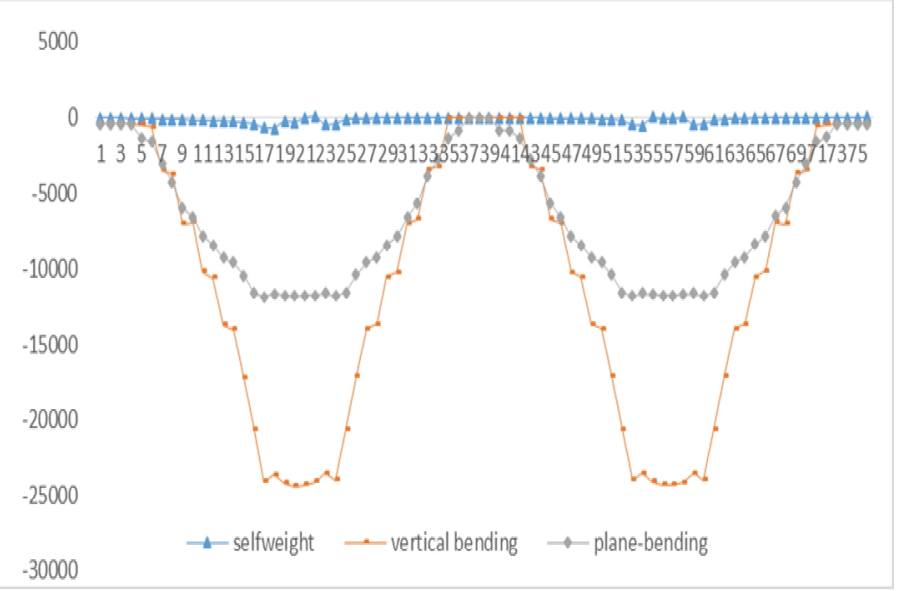

Fig 10 Transverse bending moment

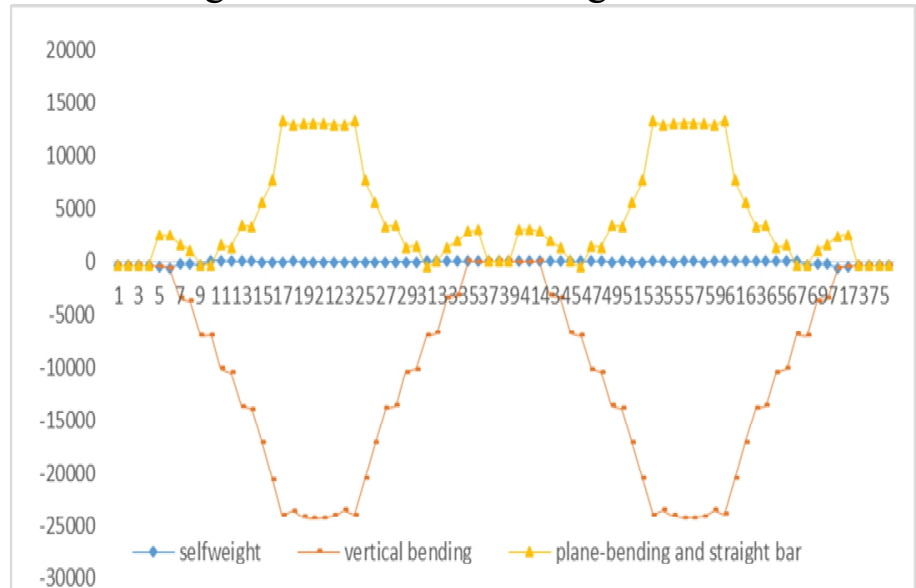

Fig11 After the adjustment of the transverse bending moment 


\section{Acknowledgment}

- This work is supported by 2014 industry support research project, funded by Guangdong Province highway Administration, Project number: 2014-11.

\section{References}

[1] Gu, X., S.-Y. Xie, and S.-M. Jia: Analysis of parameters influence on structure of continuous curved bridge. in 3rd International Conference on Civil Engineering and Transportation, ICCET 2013, December 14, 2013 - December 15, 2013. 2014. Kunming (China: Trans Tech Publications Ltd).

[2] Wang, L. and T. Qu: Seismic response analysis of the long-span curved bridge. in 2nd International Conference on Transportation Engineering, ICTE 2009, July 25, 2009 - July 27, 2009 (2009. Chengdu, China: American Society of Civil Engineers (ASCE)).

[3] Xi, J., Q. Li, and T. Wang: Calculation of state transfer of curved bridge. in 1st International Conference on Civil Engineering, Architecture and Building Materials, CEABM 2011, June 18, 2011 - June 20, 2011 (2011. Haikou, China: Trans Tech Publications).

[4] Wang, B. and Y. Liu: Effect of radius of curvature on seismic response of curved bridge. in 2011 International Conference on Civil Engineering and Building Materials, CEBM 2011, July 29, 2011 - July 31, 2011 (2011. Kunming, China: Trans Tech Publications).

[5] Dong, J. and R. Sause: Engineering Structures, 2010. 32(1), p. 319-327. 